\title{
Optimization of a virus-induced gene silencing system and functional elucidation of MaBAM9b in postharvest banana fruits
}

juhua liu( juhua69@126.com )

Institute of Tropical Bioscience and Biotechnology https://orcid.org/0000-0002-3200-6672

Meng Li

Nanjing Agricultural University

Jing Zhang

Institute of Tropical Bioscience and Biotechnology,CATAS

Hongxia Miao

Institute of Tropical Bioscience and Biotechnology, CATAS

Jingyi Wang

Institute of Tropical Bioscience and Biotechnology, CATAS

Caihong Jia

Institute of Tropical Bioscience and Biotechnology, CATAS

Jianbin Zhang

Institute of Tropical Bioscience and Biotechnology

Zhuo Wang

Institute of Tropical Bioscience and Biotechnology

Biyu Xu

Institute of Tropical Bioscience and Biotechnology

Zhiqiang Jin

Institute of Tropical Bioscience and Biotechnology

Research

Keywords: Banana (Musa spp.), VIGS, MaBAM9b, Functional identification

Posted Date: July 8th, 2020

DOI: https://doi.org/10.21203/rs.3.rs-31259/v2

License: (c) (1) This work is licensed under a Creative Commons Attribution 4.0 International License.

Read Full License 


\section{Abstract}

Background: The genetic basis of metabolic pathways that operate during fruit ripening needs to be understood before the nutritional value of the banana can be improved. The banana is a typical starch conversion fruit, and $\beta$-amylase is a key enzyme that may play an important role in starch degradation during the ripening process. Musa acuminata $\beta$-amylase $9 b(M a B A M 9 b)$ is closely related to starch degradation. However, its exact function in starch degradation has not been demonstrated in banana. Stable genetic transformation to identify gene function is time- and energy-consuming. Thus, an efficient and rapid method is needed for functional identification. Virus-induced gene silencing (VIGS) is a reversegenetics method based on RNA-mediated antiviral plant defense that has been used to rapidly identify gene functions in plants. The aim of this study is to optimize a VIGS system and functional elucidation of $M a B A M 9 b$ in postharvest banana fruits.

Results: Using $0.5 \%$ iodine-potassium-iodide $\left(\mathrm{I}_{2}-\mathrm{KI}\right)$ staining for $150 \mathrm{~s}$, we found that $1: 3$ TRV1:TRV2MaBAM9b cultivated at $30 \mathrm{mmHg}$ for $30 \mathrm{~s}$ to an optical density (OD) of 0.8 at $600 \mathrm{~nm}$, and kept on Murashige \& Skoog (MS) media for 5 days produced the best silencing results. Under these conditions, the total starch content was greatly increased, whereas the $\beta$-amylase activity, soluble sugar content, and expression of endogenous MaBAM9b greatly decreased.

Conclusions: The system described here is particularly useful for studying genes and networks involved in starch conversion in fruit, which alone would not produce a visual phenotype. This system will provide a platform for functional genomics and fruit quality improvement in the banana.

\section{Background}

Banana is an important and pleasantly flavored tropical fruit that is popular with consumers worldwide [1]. As a typical starch conversion fruit, the starch content of banana fruit upon harvesting can reach 12$35 \%$ fresh weight (FW) [2]. Starch is degraded and converted to sucrose rapidly during the post-harvest banana fruit ripening process [3]. The soluble sugars may reach up to $20 \% \mathrm{FW}$ of the pulp in the ripe fruit, with sucrose accounting for approximately $80 \%$, whereas glucose and fructose make up almost all the remaining $20 \%$ of the soluble sugars in equal proportions[2]. During banana fruit ripening process, the sucrose content varied from 12.2 to $411.8 \mathrm{mg} \mathrm{g}^{-1} \mathrm{FW}$, while the glucose and fructose contents varied from 2.1 to $100.5 \mathrm{mg} \mathrm{g}^{-1} \mathrm{FW}$ and 1.5 to $103.8 \mathrm{mg} \mathrm{g}^{-1} \mathrm{FW}$, respectively (not published data). The conversion efficiency directly decides the fruit sweetness, firmness, flavor, nutritional quality, and shelf life. Therefore, banana is a model fruit for investigating fruit starch metabolism, $\beta$-amylase is a key enzyme that converts starch to glucose [4]. Musa acuminata $\beta$-amylase 9b (MaBAM9b, Ma05_t07800.1) is highly expressed during banana fruit ripening and is closely related to starch degradation [5-6]. However, its exact function in starch degradation has not been demonstrated in banana. Elucidating the function of $M a B A M 9 b$ in banana using genetic transformation could take in excess of three years due to various banana-specific challenges such as slow growth process, low transgenic efficiency and high dependence 
on genotype [7]. Therefore, developing an efficient way to rapidly and exactly identify gene function is important for banana functional genomics.

Virus-induced gene silencing (VIGS) is a reverse-genetics tool that has powerful applications in functional genomics [8]. In VIGS, the recombinant virus carrying a plant cDNA fragment infects the plant tissue and spreads systemically throughout the tissue. Then, the endogenous gene transcripts, which are homologous to the insert fragment in the viral vector, are degraded by post-transcriptional gene silencing (PTGS). Finally, the gene function can be assessed based on the plant phenotype and physiological index alteration [9]. VIGS can be developed rapidly and has been applied to functional gene identification in fleshy fruits, such as tomato [8,10], strawberry [11-13], apple [14-15], grape [16], citrus [17], loquat [18], pear [19-20], peach [21-22] and sweet cherry [23-24]. However, there are no reports on VIGS in banana.

In this report, a VIGS-mediated banana fruit slice system was developed to rapidly elucidate the role of $M a B A M 9 b$ in banana fruit starch metabolism. The effects of different ratios of pTRV2-MaBAM9b with pTRV1, the OD600 values of the bacterial fluid, and the co-cultivation times on the silencing results were carefully investigated. The results showed that the co-cultivation of 2-4-mm-thick fruit slices at a 1:3 ratio of pTRV1: pTRV2-MaBAM9b at OD600 0.8 at $30 \mathrm{mmHg}$ for $30 \mathrm{sec}$ and kept on Murashige \& Skoog (MS) media for $5 \mathrm{~d}$ could obtain the most efficient silencing results based on an analysis of the contents of total starch, glucose, $\beta$-amylase activity, and MaBAM $9 b$ expression. These results will provide a platform for banana functional genomics on starch metabolism and should contribute towards developing a technique for controlling starch degradation.

\section{Results}

\section{The effects of different $\mathrm{I}_{2}-\mathrm{KI}$ solutions and times on the staining results}

As shown in Fig. 1, as the treatment duration and concentration increased, the blue stain gradually became darker. The $0.1 \%(\mathrm{a})$ and $0.25 \%$ (b) $\mathrm{I}_{2}-\mathrm{KI}$ solutions did not complete their staining in any of the tested treatment times (Fig. $1 \mathrm{~A}-\mathrm{F}$ ). The $0.75 \%$ (d) and $1.0 \%$ (e) $\mathrm{I}_{2}-\mathrm{KI}$ solutions resulted in an uneven dark or light color under long or short treatment times, respectively. The banana fruit slices stained with $0.5 \%$ $\mathrm{I}_{2}-\mathrm{KI}$ solution for $150 \mathrm{~s}$ obtained a more uniform staining effect that allowed for easier identification.

\section{The effects of different ratios of pTRV2-MaBAM9b with pTRV1 on the silencing results}

As shown in Fig. 2A, when the ratio of pTRV2-MaBAM9b with pTRV1 reached $1: 2$, the blue stain of $\mathrm{I}_{2}-\mathrm{KI}$ became darker. The color became darkest at a ratio of 1:3 compared to the control (CK). This result indicated that ratios of $1: 2$ and 1:3 could efficiently suppress starch degradation. Further investigation found that the contents of soluble sugars, including glucose, fructose, and sucrose, were greatly decreased by $62.8 \%, 61.9 \%$, and $62.6 \%$ compared to CK, respectively, at a ratio $1: 3$ (Fig. 2B). The $\beta$ amylase activities were also significantly decreased compared to $\mathrm{CK}$, decreasing by $18.7 \%, 62.7 \%$, and $76.7 \%$ at ratios of $1: 1,1: 2$, and $1: 3$, respectively (Fig. 2 C). In contrast, the total starch contents at ratios of 
$1: 1,1: 2$, and $1: 3$ were $339.7,398.8$, and 436.2 , indicating a $2.7 \%, 20.5 \%$, and $31.9 \%$ increase compared to $\mathrm{CK}$, respectively (Fig. 2D). The expression of endogenous $M a B A M 9 \mathrm{~b}$ was greatly suppressed, decreasing by $28.0 \%, 42.0 \%$, and $63.0 \%$ at ratios of $1: 1,1: 2$, and $1: 3$, respectively. These results indicated that the ratio of pTRV2-MaBAM9b together with pTRV1 at 1:3 obtained the best silencing efficiency.

\section{The effects of different bacterial fluid OD values on the silencing results}

As shown in Fig. 3A, with the increase in OD600 value of the bacterial fluid from 0.4 to 0.6 , the $\mathrm{I}_{2}-\mathrm{KI}$ staining gradually became darker compared to $\mathrm{CK}$. When the OD600 value reached 0.8 , the stain was darkest. However, when the OD600 value increased to 1.0 or 1.2, the staining became weaker. This result demonstrated that an OD600 of 0.8 obtained the best results for suppressing starch degradation. Further investigation indicated that the total starch contents at OD600 values of $0.4,0.6,0.8,1.0$, and 1.2 were $235.7,264.5,326.0,303.1$, and 278.4 , which were increased by $6.9 \%, 19.7 \%, 47.6 \%, 37.2 \%$, and $26.0 \%$ relative to CK, respectively (Fig. 3B). Conversely, the $\beta$-amylase activities at OD600 values of $0.4,0.6,0.8$, 1.0 , and 1.2 were significantly decreased compared to $\mathrm{CK}$, decreasing by $9.2 \%, 15.3 \%, 27.6 \%, 22.4 \%$, and $9.2 \%$, respectively (Fig. $3 \mathrm{C}$ ). The contents of soluble sugars, including glucose, fructose, and sucrose, were also greatly reduced by $67.2 \%, 67.8 \%$, and $74.8 \%$ compared to CK, respectively, at OD600 0.8 (Fig. 3D). The expression of endogenous MaBAM9b was greatly suppressed, decreasing by $29.0 \%, 49.0 \%$, $79.0 \%, 68.0 \%$, and $52.0 \%$, respectively. These results suggested that an OD600 value of 0.8 obtained the best silencing efficiency.

\section{The effects of different co-cultivation times on the silencing results}

As shown in Fig. 4A, as the co-cultivation time progressed, the blue stain of $\mathrm{I}_{2}-\mathrm{KI}$ became gradually darker. When the co-cultivation time reached $5 \mathrm{~d}$, the color was the darkest. However, when the co-cultivation time increased to $7 \mathrm{~d}$, the staining became weaker. This result demonstrated that co-cultivation for $5 \mathrm{~d}$ was best for suppressing starch degradation. Further investigation found that the total starch contents at $3 \mathrm{~d}$, $5 \mathrm{~d}$, and $7 \mathrm{~d}$ were $198.4,315.7$, and 301.4 , increasing by $12.5 \%, 79.1 \%$, and $71.0 \%$ compared to $1 \mathrm{~d}$, respectively (Fig. 4B). Conversely, the $\beta$-amylase activities at co-cultivation times of $3 d, 5 d$, and $7 d$ were significantly reduced compared to $1 \mathrm{~d}$, decreasing by $10.7 \%, 29.8 \%$, and $27.4 \%$, respectively (Fig. $4 \mathrm{C}$ ). The contents of glucose, fructose, and sucrose also decreased greatly by $60.5 \%, 53.8 \%$, and $64.1 \%$ at an cocultivation time of $5 \mathrm{~d}$, respectively (Fig. 4D). The expression of endogenous MaBAM9b was greatly suppressed by $26.0 \%, 58.0 \%$, and $42.0 \%$, respectively. These results suggested that $5 \mathrm{~d}$ of co-cultivation obtained the best silencing efficiency.

\section{Discussion}

Banana is an important global tropical climacteric fruit with high nutritional value. Fruit ripening involves a quality formation process, during which many irreversible physiological and biochemical changes occur. Starch is the main component and the building block of banana fruit. Starch conversion to sugar is a primary metabolic process that directly influences the fruit quality, such as the texture, flavor, and shelf 
life [3]. Understanding the molecular mechanism of fruit starch degradation metabolism is necessary for improving fruit quality. $M a B A M 9 b$ is a key enzyme gene that might play an important role in starch degradation during the banana fruit ripening process [5]. However, its function has not been explicitly identified in banana. Identifying gene functions using stable genetic transformation is time- and energyconsuming. It is thus necessary that an efficient means for rapid functional identification is developed. VIGS is a reverse-genetics method based on RNA-mediated antiviral plant defense that has been used to rapidly identify gene function in plants [25]. The efficiency of VIGS in inducing endogenous gene silencing is related to multiple factors, such as the correct viral vectors, the characteristics of the target gene, the infection buffer, the activity of Agrobacterium in the bacterial suspension, and the plant growth environment suitable for silencing [26]. Using MaBAM9b as an example, we optimized the VIGS system to facilitate gene function analysis.

The selection of experimental material is the first important step for VIGS. In sweet cherry, Li et al. (2015) [23] selected 28 days after full bloom (DAFB) fruits as material for TRV vector injection. In Hanfu' apple, pear, and loquat, 15 DAFB, 30 DAFB, and breaker stage fruits were used as materials for TRV vector injection, respectively [14,18-19]. We selected 8 days postharvest (DPH) fruits to investigate the function of MaBAM $9 b$ by VIGS, as $8 \mathrm{DPH}$ is the key stage of rapid starch degradation, as has been demonstrated by Wang et al. (2019) [6].

$\mathrm{I}_{2}$ - $\mathrm{KI}$ staining is a visual technique for quickly identifying starch contents in plant organs. This technique has been widely applied in rice [27], Zea mays [28], and Arabidopsis [29]. The result of $\mathrm{I}_{2}-\mathrm{KI}$ staining is influenced by factors such as staining solution and time. However, these factors have not been optimized in banana. Here, we investigated the effects of the concentration of the staining solution and the staining duration on the staining efficiency. The results indicated that 2-4 mm-thick banana fruit slices stained with $0.5 \% \mathrm{I}_{2}-\mathrm{KI}$ solution for $150 \mathrm{~s}$ obtained the best staining effect. This result provides a reference for the rapid identification of starch contents, especially for high-starch fruits.

Successful gene silencing depends upon a dynamic interplay between virus spread and plant growth, both of which can be influenced by various conditions [26]. Different fruits have different optimized conditions for VIGS. For Lycium plants, the optimized conditions included an OD600 of 1.0, with the cells incubated at room temperature for 3-4 $\mathrm{h}$. The pTRV1 and pTRV2 solutions were mixed at a ratio of 1:1 for injection [30]. In tomato, the OD600 was adjusted to 0.5 or 1.0 for all of the vectors, and the resuspended cells with pTRV1 and pTRV2 were then combined at a ratio of 1:1 and kept for more than 10 $\mathrm{d}$ in a plastic tray $[8,31]$. In loquat, the OD600 was adjusted to 1.2 , and the resuspended cells with pTRV1 and pTRV2 were combined at a ratio of 1:1 and co-cultivated for a week [18]. In the present study, the effects of different ratios of pTRV2-MaBAM9b and pTRV1, OD600 values, and co-cultivation durations on the silencing results were systematically investigated. The results showed that a 1:3 ratio of pTRV1: pTRV2-MaBAM9b, an OD600 of 0.8 , and $5 \mathrm{~d}$ of cultivation obtained the most efficient silencing results. This system was something different from other fruits and reflected the distinguishing qualities of banana fruit. 


\section{Conclusion}

A TRV-mediated VIGS system was successfully constructed and used to efficiently silence MaBAM9b, which resulted in increased total starch contents and decreased $\beta$-amylase activity, soluble sugar content, and endogenous MaBAM9b expression. These findings support that MaBAM9b plays a vital role in banana fruit starch degradation metabolism, thus providing a platform for banana functional genomics and for the breeding of new banana varieties.

\section{Methods}

\section{Plant materials}

Banana (Musa AAA group cv Brazilian) fruits were harvested from the banana plantation of the Institute of Tropical Bioscience and Biotechnology (Chengmai, Hainan, 20N, 110E) and transported back to the laboratory. The healthy middle fruit comb was selected and separated into a single fruit finger. After surface-sterilization for 10 min with $0.1 \%$ sodium hypochlorite, the fruit fingers were air-dried and stored for $8 \mathrm{~d}$ at $22^{\circ} \mathrm{C}$ and $80 \%$ relative humidity.

A sharp blade that has been surface-sterilized with $75 \%$ ethanol was used to uniformly section the fruit fingers into 2-4-mm-thick slices, with the fruit ends discarded.

\section{lodine-potassium-iodide $\left(\mathrm{I}_{2}-\mathrm{KI}\right)$ staining and treatments}

The banana fruit slices were immersed in $0.1 \%, 0.25 \%, 0.5 \%, 0.75 \%$, and $1.0 \% \mathrm{I}_{2}-\mathrm{KI}$ solutions for $30 \mathrm{~s}, 60 \mathrm{~s}$, $90 \mathrm{~s}, 120 \mathrm{~s}, 150 \mathrm{~s}$, and $180 \mathrm{~s}$. The effect of dyeing was assessed based on the intensity of the stain.

\section{Plasmid construction}

The tobacco rattle virus (TRV)-based VIGS vectors pTRV1 and pTRV2 (described in Liu et al., 2002) [32] were kindly offered by $\mathrm{Dr} \mathrm{H}$. Shi from Hainan University. A 586-bp gene fragment corresponding to bases 912-1498 of MaBAM9b (Ma05_t07800.1) was PCR-amplified from banana cDNA templates using primers (forward: 5'-GGTCTAGATCCGGACTTCGGCAACTTT-3' with an Xba I restriction site and reverse: 5'CGGGATCCCGCCATGCTCCTGATGAAC-3' with an BamH I restriction site). The resulting product was cloned into pTRV2 to form pTRV2- MaBAM9b.

\section{VIGS in the banana fruit slices}

The Agrobacterium tumefaciens GV3101 cell cultures harboring pTRV2 and pTRV2- MaBAM9b with pTRV1 were mixed at ratios of $1: 1,1: 2$, and $1: 3$, and the resuspended $A$. tumefaciens $\mathrm{GV} 3101$ cells were cultivated at OD600 values of $0.4,0.6,0.8,1.0$, and 1.2. The banana fruit slices were vacuum-infiltrated with the Agrobacterium solution at $30 \mathrm{mmHg}$ for $30 \mathrm{~s}$. After the release of the vacuum, the fruit slices were maintained for $1 \mathrm{~d}, 3 \mathrm{~d}, 5 \mathrm{~d}$, and $7 \mathrm{~d}$ on Murashige \& Skoog (MS) medium at $23^{\circ} \mathrm{C}$. The banana fruit slices at each point were sampled for $\mathrm{I}_{2}-\mathrm{Kl}$ staining, gene expression, and physiological index analysis. 
The total RNA in the banana fruit slices was extracted using an RNAprep Pure Plant Kit (Tiangen, DP441, Beijing, China) together with RNase free DNase (NEB, M0303S, USA) according to the manufacturer's protocol. First-strand cDNA was synthesized using a RevertAid First Strand cDNA Synthesis Kit and 20 $\mu \mathrm{L}$ was further diluted in 1:50 and quantified using a Nanodrop 2000 (both Thermo Scientific, Waltham, MA, USA). The changes in the transcriptome of MaBAM9b were evaluated by qRT-PCR analysis on a Stratagene Mx3000P Real-Time PCR system with SYBR® Premix Ex Taq ${ }^{\text {TM }}$ (TaKaRa, Japan) using the 2${ }^{\triangle \triangle} \mathrm{Ct}$ method [33]. MaRPS2 (HQ853246) and MaUBQ2 (HQ853254) constituted the internal controls that were used to normalize the target gene expression [34]. The primers for MaBAM9b expression are as follows: F: 5-GCCCGCAGTCACTACTATCT-3, R: 5-GAAGAA CTCAGCA CCCATCC-3.

\section{Quantification of physiological index}

The total starch content was detected following the method of Miao et al. (2014) [35]. The $\beta$-amylase activity was measured according to the method of Hou et al. (2017) [36]. The soluble sugar (fructose, glucose and sucrose) contents were analyzed using High-Performance Liquid Chromatography (HPLC; Waters, Milford, CT, USA). The HPLC method was developed and validated using an AMINEX 87H column, $10 \mathrm{mmol} \mathrm{L}^{-1}$ sulfuric acid as eluent and refraction index detection[37].

\section{Statistical analysis}

At least three biological replicates were assessed, and all of the data were analyzed using One-way analysis of variance (ANOVA) and Student's $t$-tests for determination of significant differences.

\section{Declarations}

\section{Ethics approval and consent to participate}

Not applicable.

\section{Consent for publication}

Not applicable.

\section{Availability of data and materials}

All data generated or analysed during this study are included in this published article.

\section{Competing interests}

The authors declare that they have no competing interests.

\section{Funding}


The work was sponsored by the National Natural Science Foundation of China (NNSFC, No.31872161), the National Key R \& D Program of China (No.2018YFD1000200 and 2019YFD1000200), the Central Public-interest Scientific Institution Basal Research Fund for Innovative Research Team Program of CATAS (No.1630052017018; 1630052019024) and the Modern Agro-industry Technology Research System of China (No. CARS-31).

\section{Authors' contributions}

$\mathrm{LJH}, \mathrm{XBY}$ and JZQ conceived and designed the experiments. LJH and LM performed the experiments and analyzed the data, wrote the paper, prepared the figures, and reviewed drafts of the paper. ZJ, MHX, WJY, $\mathrm{JCH}, \mathrm{ZJB}$ and WZ executed the experimental materials and laboratory experiments, acquired image data. All authors read and approved the final manuscript.

\section{Acknowledgments}

We thank $\mathrm{DrH}$. Shi from Hainan University for his kindly offered the VIGS vectors. We thank LetPub (www.letpub.com) and San Francisco Edit (https://sfedit.net) for their linguistic and scientific assistance during the preparation of this manuscript.

\section{Abbreviations}

ANOVA, analysis of variance; DAFB, days after full bloom; DPH, days postharvest; FW, fresh weight; $\mathrm{I}_{2}-\mathrm{KI}$, iodine-potassium-iodide; MaBAM9b,Musa acuminate $\beta$-amylase 9b; MS, Murashige \& Skoog; OD, optical density; PTGS, post-transcriptional gene silencing; qRT, quantitative real-time; TRV, tobacco rattle virus; VIGS, virus-induced gene silencing.

\section{References}

1. Bapat VA, Trivedi PK, Ghosh A, Sane VA, Ganapathi TR, Nath P. Ripening of fleshy fruit: molecular insight and the role of ethylene. Biotechnol Advances. 2010; 28: 94-107.

2. Cordenunsi-Lysenko BR, Nascimento JRO, Castro-Alves VC, Purgatto E, Fabi JP, Peroni-Okyta FHG. The starch is (not) just another brick in the wall: the primary metabolism of sugars during banana ripening. Front Plant Sci. 2019; 10:391.

3. Xiao YY, Kuang JF, Qi XN, Ye YJ, Wu ZX, Chen JY, et al. A comprehensive investigation of starch degradation process and identification of a transcriptional activator MabHLH6 during banana fruit ripening. Plant Biotechnol J. 2018; 16: 151-164.

4. do Nascimento JRO, Junior AV, Bassinello PZ, Cordenunsi BR, Mainardi JA, Purgatto E, et al. Betaamylase expression and starch degradation during banana ripening. Postharvest Biol Technol. 2006; 40: 41-47.

5. Miao HX, Sun PG, Miao YL, Liu JH, Zhang JB, Jia CH, et al. Genome-wide identification and expression analysis of the $\beta$-amylase genes strongly associated with fruit development, ripening, and 
abiotic stress response in two banana cultivars. Front Agr Sci Eng. 2016; 3: 346-356.

6. Wang Z, Miao HX, Liu JH, Xu BY, Yao XM, Xu CY, et al. Musa balbisiana genome reveals subgenome evolution and functional divergence. Nature Plants. 2019; 5: 810-821.

7. Liu JH, Gao PZ, Sun XX, Zhang J, Sun PG, Wang JS, et al. Efficient regeneration and genetic transformation platform applicable to five Musa varieties. Electronic J Biotechnol. 2017; 25: 33-38.

8. Fu DQ, Zhu BZ, Zhu HL, Jiang WB, Luo YB.Virus-induced gene silencing in tomato fruit. Plant J.2005; 43: 299-308.

9. Baulcombe DC. RNA silencing in plants. Nature. 2004; 431: 356-363.

10. Naing AH, Kyu SY, Pe PPW, Park KI, Lee JM, Lim KB, et al.Silencing of the phytoene desaturase (PDS) gene affects the expression of fruit-ripening genes in tomatoes. Plant Methods.2019; 15: 110.

11. Chen SY, Tang YM, Hu YY, Wang Y, Sun B, Wang XR, et al. FaTT12-1, a multidrug and toxin extrusion (MATE) member involved in proanthocyanidin transport in strawberry fruits. Sci Hortic. 2018; 231: 158-165.

12. Li C, Yamagishi N, Kasajima I, Yoshikawa N. Virus-induced gene silencing and virus-induced flowering in strawberry (Fragariaxananassa) using apple latent spherical virus vectors. Hortic Res.2019; 6:18.

13. Xie YG, Ma YY, Bi PP, Wei W, Liu J, Hu Y, et al. Transcription factor FvTCP9 promotes strawberry fruit ripening by regulating the biosynthesis of abscisic acid and anthocyanins. Plant Physiol Biochem.2020; 146: 374-383.

14. Li T, Tan D, Liu Z, Jiang Z, Wei Y, Zhang L, et al. Apple MdACS6 regulates ethylene biosynthesis during fruit development involving ethylene-responsive factor. Plant Cell Physiol.2015; 56:1909-17.

15. Jia M, Du P, Ding N, Zhang Q, Xing S, Wei L, et al. Two FERONIA-like receptor kinases regulate apple fruit ripening by modulating ethylene production. Front Plant Sci.2017; 8:1406.

16. Gao Z, Li Q, Li J, Chen Y, Luo M, Li H, et al. Characterization of the ABA receptor VIPYL1 that regulates anthocyanin accumulation in grape berry skin. Front Plant Sci.2018; 9:592.

17. Killiny N, Hijaz F, Nehela Y, Hajeri S, Gowda S. Effects of $\delta$ - aminolevulinic acid dehydratase silencing on the primary and secondary metabolisms of citrus. Plant Direct.2018; 2: e00072.

18. Hong M, Chi ZH, Wang YQ, Tang YM, Deng QX, He MY, et al. Expression of a chromoplast-specific lycopene $\beta$-cyclase gene $(C Y C-B)$ is implicated in carotenoid accumulation and coloration in the loquat. Biomolecules.2019; 9: pii: E874.

19. Zhai R, Wang Z, Zhang S, Meng G, Song L, Wang Z, et al. Two MYB transcription factors regulate flavonoid biosynthesis in pear fruit (Pyrus bretschneideri Rehd.). J Exp Bot. 2015; 67: 1275-1284.

20. Hao L, Zhang Y, Wang S, Zhang W, Wang S, Xu C, et al. A constitutive and drought-responsive mRNA undergoes long-distance transport in pear (Pyrus betulaefolia) phloem. Plant Sci.2020; 293:110419.

21. Li J, Li F, Qian M, Han M, Liu H, Zhang D, et al. Characteristics and regulatory pathway of the PrupeSEP1 SEPALLATA gene during ripening and softening in peach fruits. Plant Sci.2017; 257: 6373. 
22. Liu H, Qian M, Song C, Li J, Zhao C, Li G, et al. Down-regulation of $P p B G A L 10$ and $P p B G A L 16$ delays fruit softening in peach by reducing polygalacturonase and pectin methylesterase activity. Front Plant Sci.2018; 9: 1015.

23. Li Q, Chen P, Dai SJ, Sun YF, Yuan B, Kai WB, et al. PacCYP707A2 negatively regulates cherry fruit ripening while PacCYP707A1 mediates drought tolerance. J Exp Bot. 2015; 66: 3765-3774.

24. Qi X, Liu C, Song L, Li Y, Li M. PaCYP78A9, a Cytochrome P450, regulates fruit size in sweet cherry (Prunus aviumL.). Front Plant Sci.2017; 8: 2076.

25. Wang C, Fu D. Virus-induced gene silencing of the eggplant chalcone synthase gene during fruit ripening modifies epidermal cells and gravitropism. J Agric Food Chem.2018; 66: 2623-2629.

26. Burch-Smith TM, Anderson JC, Martin GB, Dinesh-Kumar SP. Applications and advantages of virusinduced gene silencing for gene function studies in plants. Plant J. 2004; 39: 734-746.

27. Xing S, Meng X, Zhou L, Mujahid H, Zhao C, Zhang Y, et al. Proteome profile of starch granules purified from rice (Oryza sativa) endosperm. PLoS One.2016; 11: e0168467.

28. Jiang L, Jing GX, Li XY, Wang XQ, Xing Z, Deng PK, et al. Tissue culture characteristics of maize (Zea mays L.) haploid coleoptile sections. Genet Mol Res.2015; 14:16265-75.

29. Hedhly A, Vogler H, Schmid MW, Pazmino D, Gagliardini V, Santelia D, et al. Starch turnover and metabolism during flower and early embryo development. Plant Physiol.2016; 172: 2388-2402.

30. Li G, Zhao JH, Qin BB, Yin Y, An W, Mu ZX, et al. ABA mediates development-dependent anthocyanin biosynthesis and fruit coloration in Lycium plants. BMC Plant Biol. 2019; 19: 317.

31. Meng L, Fan Z, Zhang Q, Wang C, Gao Y, Deng Y, et al. BEL1- LIKE HOMEODOMAIN 11 regulates chloroplast development and chlorophyll synthesis in tomato fruit. Plant J. 2018; 94: 1126-1140.

32. Liu Y, Schiff M, Dinesh-Kumar SP. Virus-induced gene silencing in tomato. Plant J. 2002; 31: 777-786.

33. Livak KJ, Schmittgen TD. Analysis of relative gene expression data using real-time quantitative PCR and the 2- $\triangle \triangle \mathrm{CT}$ method. Methods. 2001; 25: 402-408.

34. Chen L, Zhong HY, Kuang JF, Li JG, Lu WJ, Chen JY. Validation of reference genes for RT-qPCR studies of gene expression in banana fruit under different experimental conditions. Planta. 2011; 234: $377-390$.

35. Miao HX, Sun PG, Liu WX, Xu BY, Jin ZQ. Identification of genes encoding granule-bound starch synthase involved in amylose metabolism in banana fruit. PLoS One. 2014; 9: e88077.

36. Hou J, Zhang H, Liu J, Reid S, Liu T, Xu S, Tian Z, Sonnewald U, Song B, Xie C. Amylases StAmy23, StBAM1 and StBAM9 regulate cold-induced sweetening of potato tubers in distinct ways. J Exp Bot. 2017; 68: 2317-2331.

37. Duarte-Delgado D, Narváez-Cuenca CE, Restrepo-Sánchez LP, Kusha-lappa A, Mosquera-Vásquez T. Development and validation of a liquid chromatographic method to quantify sucrose, glucose, and fructose in tubers of Solanum tuberosum Group Phureja. J Chromatogr B. 2015; 975:18-23. 


\section{Figures}
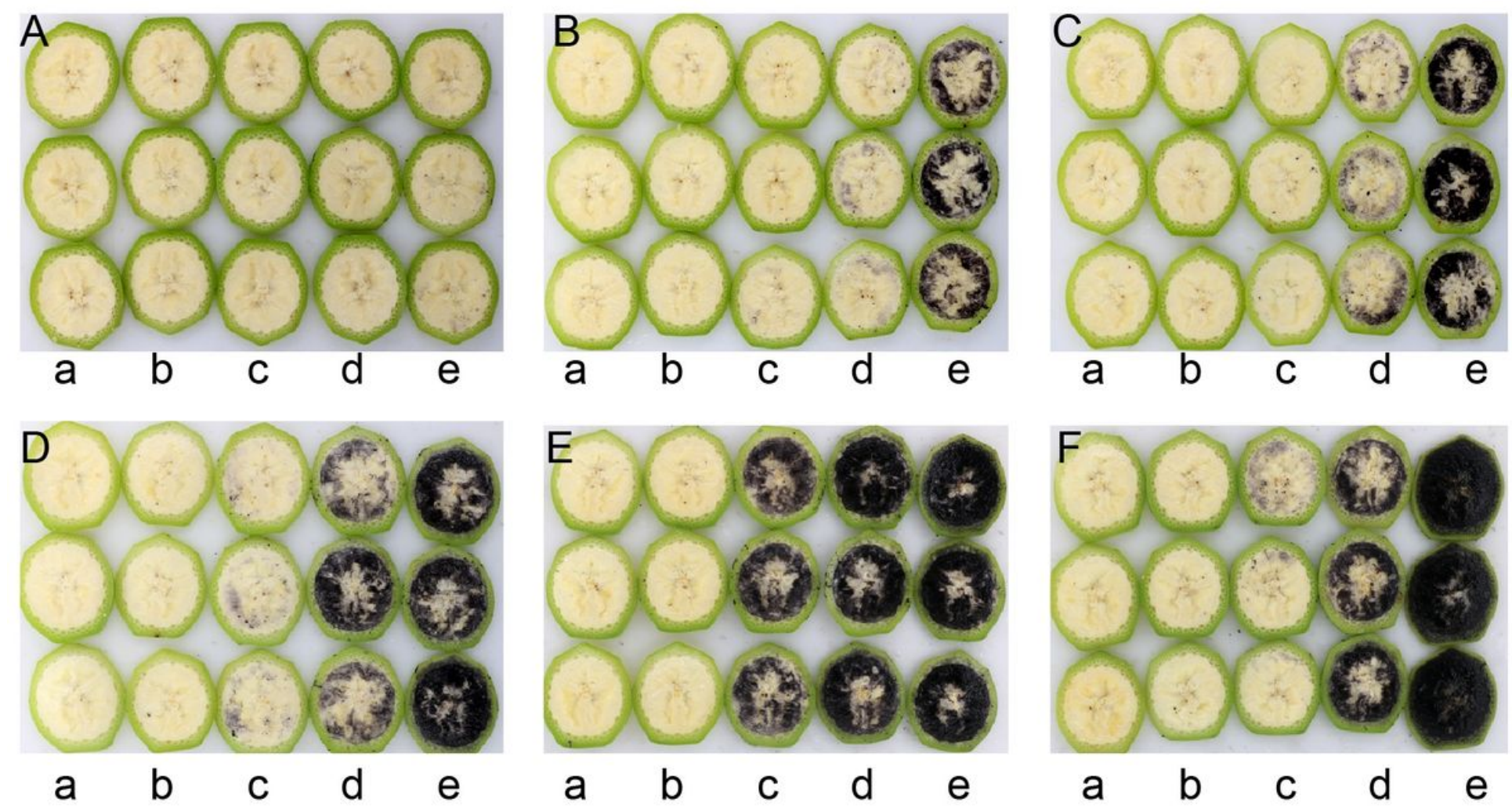

\section{Figure 1}

The effects of different I2-KI solution concentrations and treatment times on the staining results. A-F, staining for $30 \mathrm{~s}, 60 \mathrm{~s}, 90 \mathrm{~s}, 120 \mathrm{~s}, 150 \mathrm{~s}$, and $180 \mathrm{~s}$, respectively; a-e, I2-KI solutions of $0.1 \%, 0.25 \%, 0.5 \%$, $0.75 \%$, and $1.0 \%$, respectively 
A

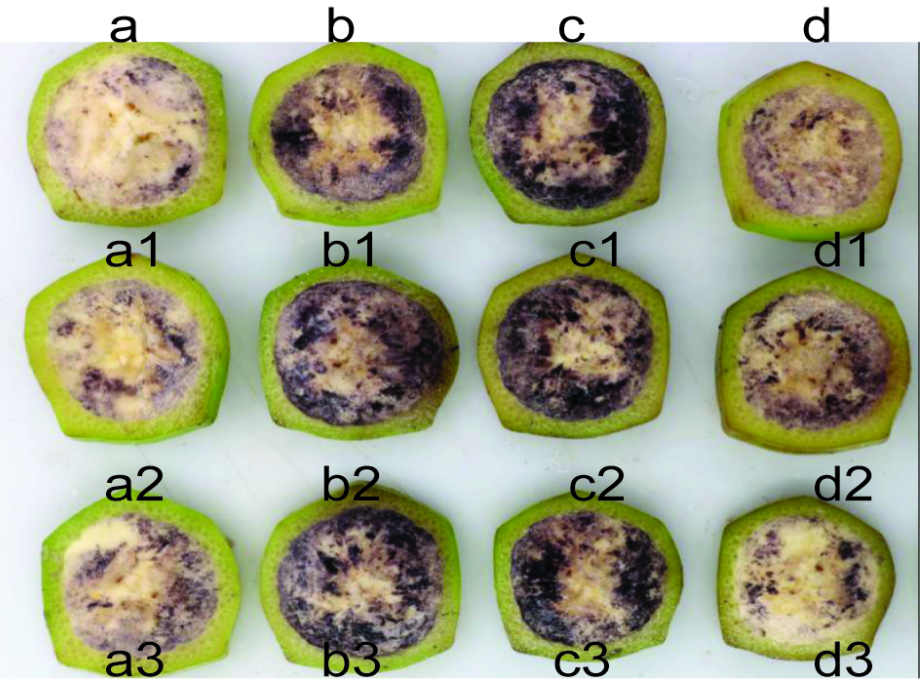

C
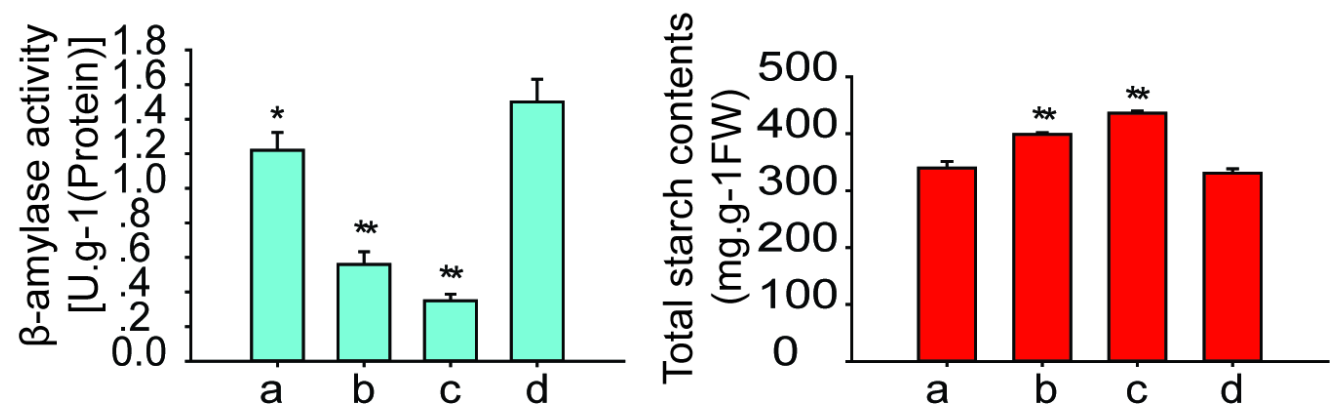

B

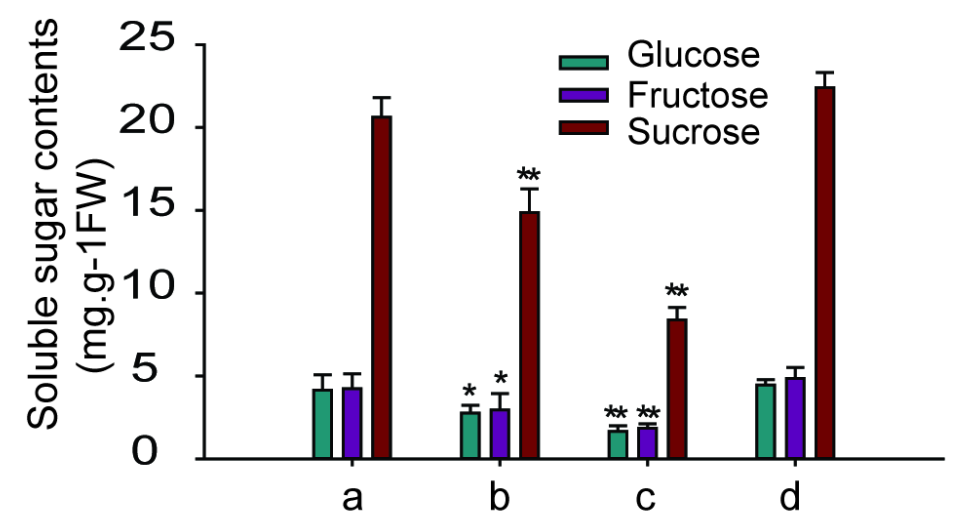

$E$

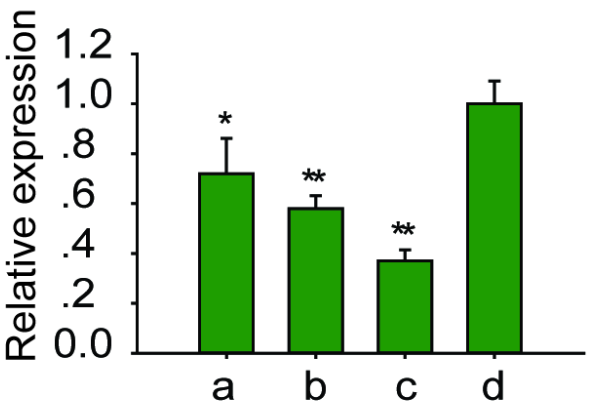

Figure 2

The effects of different ratios of pTRV1: pTRV2-MaBAM9b on the silencing results. A, the effects of I2-KI staining; $B$, soluble sugar contents; $C$, $\beta$-amylase activity; $D$, total contents of starch; $E$, expression of MaBAM9b. a-c, the ratios of 1:1,1:2, and 1:3, respectively. d, empty TRV2 plasmid as a negative control. The a1-a3, b1-b3, c2-c3, and d1-d3 represent three repeats for a, b, c, and d, respectively. The statistical significance of the differences was assessed by ANOVA $(*, p<0.05 ; * \star, p<0.01)$. 

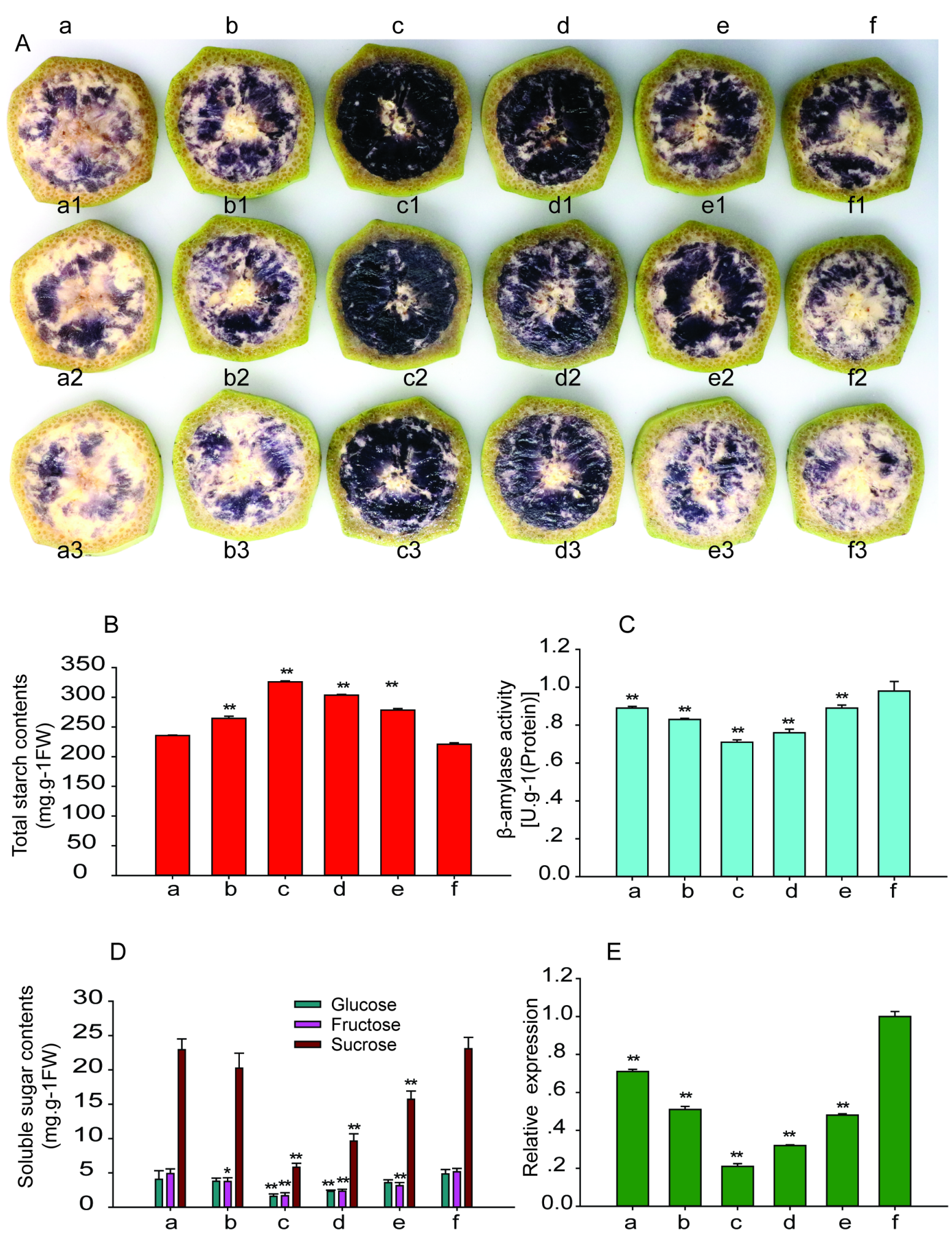

\section{Figure 3}

The effects of different OD values of the bacterial fluid on the silencing results. A, the effects of I2-KI staining; $B$, total contents of starch; $C, \beta$-amylase activity; $D$, soluble sugar contents; $E$, expression of MaBAM9b. a-e, the OD value of $0.4,0.6,0.8,1.0$, and 1.2 , respectively. $f$, empty TRV2 plasmid as a negative control. The a1-a3, b1-b3, c1-c3, d1-d3, e1-e3, and f1-f3 represent three repeats for a, b, c, d, e, 
and $f$, respectively. The statistical significance of the differences was assessed by ANOVA $(*, p<0.05 ; \star \star$, $p<0.01)$.
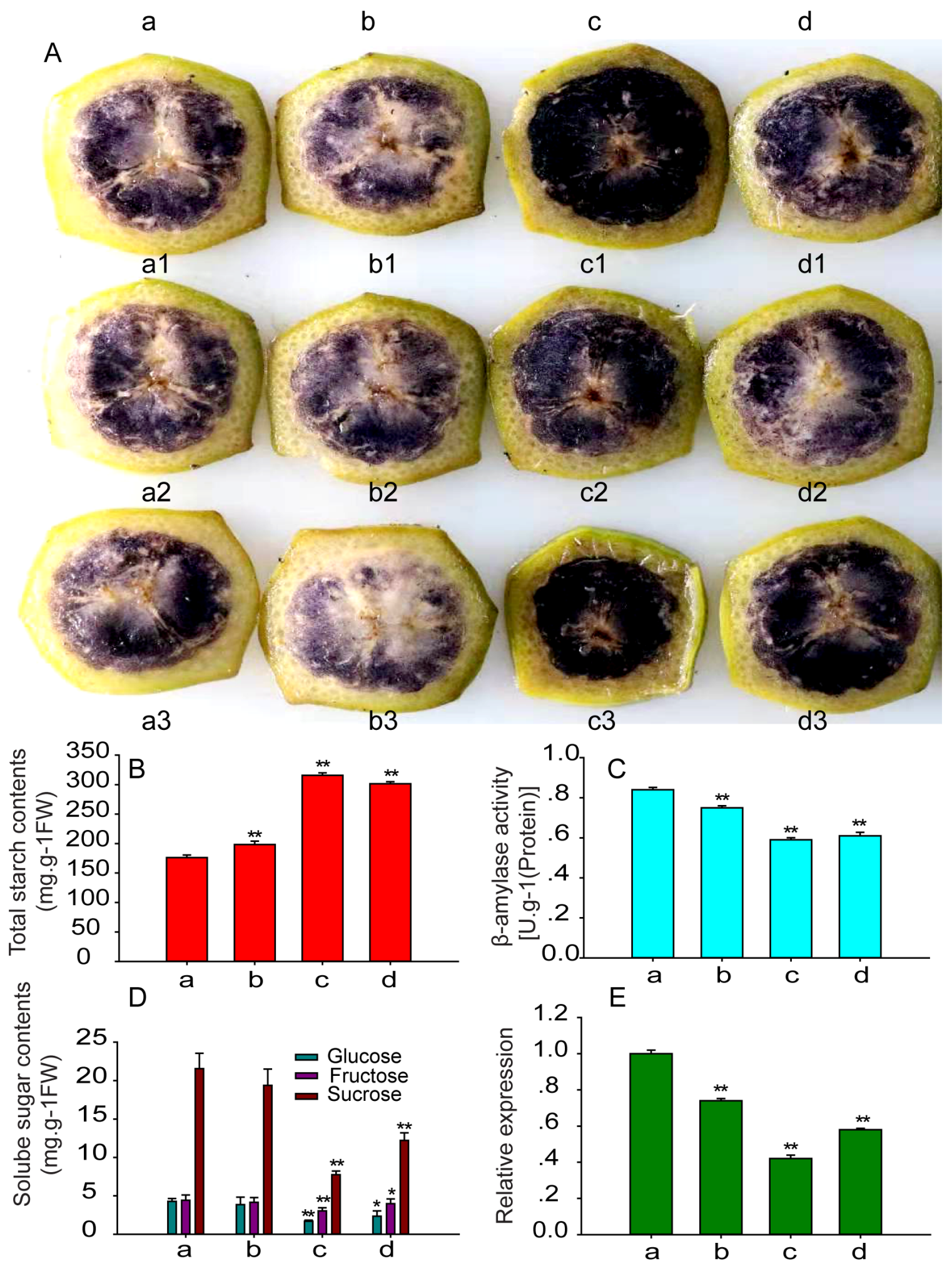

Figure 4

The effects of co-cultivation time on the silencing results. A, the effects of I2-KI staining; $\mathrm{B}$, total contents of starch; $C, \beta$-amylase activity; $D$, soluble sugar contents; $E$, expression of MaBAM9b. $a-d$, the cocultivation time for $1 \mathrm{~d}, 3 \mathrm{~d}, 5 \mathrm{~d}$, and $7 \mathrm{~d}$, respectively. The a1-a3, b1-b3, c2-c3, and d1-d3 represent three 
repeats for a, b, c, and d, respectively. The statistical significance of the differences was assessed by ANOVA $(*, p<0.05 ; * *, p<0.01)$. 